DOI: $10.20472 /$ IAC.2019.049.041

\title{
AKIKO WATANABE
}

Aoyama Gakuin University, Japan

\section{YOSUKE KURIHARA}

Aoyama Gakuin University, Japan

\section{SATOSHI KUMAGAI}

Aoyama Gakuin University, Japan

\section{TOSHIYUKI MATSUMOTO}

Aoyama Gakuin University, Japan

\section{TAKUYA HIDA}

Aoyama Gakuin University, Japan

\section{METHOD FOR DISCRIMINATING THE MODE OF OPERATION OF THE STEERING WHEEL DURING CAR DRIVING}

\begin{abstract}
:
Most traffic accidents in Japan are caused by a lack of attention by drivers. One of the main factors contributing to this is "driving fatigue". Previous research based on electromyographic evaluations has indicated that the optimal driving methods for reducing the upper limb load on drivers are an "underside" grip position and the "push-pull" steering operation technique. This previous research developed the systems that estimate the upper limb load during car driving. However, the systems developed only estimate the upper limb load based on a driving route assumed before driving a car: the problem being that actual driving operation is not considered. Therefore, we previously proposed a method for discriminating the grip position when steering on a straight road to estimate the upper limb load based on steering wheel operation during actual driving. This study now proposes a method for discriminating the modes of steering operation during turns to the right and left. First, we conduct an experiment with a motion sensor and a simple driving simulator to detect the two modes of steering-wheel operation (hand-over-hand steering and push-pull steering) on turning a corner. The 10 subjects ( 5 males and 5 females) perform the steering operation for each mode according to driving videos. It is found that the modes of steering operation can be discriminated because there are differences in the measurement results of the motion sensor for the two modes Second, using the experimental results, the method of steering operation is discriminated through machine learning with a support vector machine (SVM), supplying the SVM with the average value, standard deviation, maximum value, and minimum value of the measurements taken during steering operation. The discrimination accuracy rate of the test data is found by 5 -fold cross-validation to be 97.5\%.
\end{abstract}

\section{Keywords:}

steering operation, upper limb load, support vector machine

JEL Classification: 119 